\title{
Reliability Analysis of Bus Timetabling Strategy during the COVID-19 Epidemic: A Case Study of Yixing, China
}

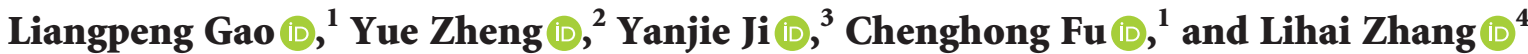 \\ ${ }^{1}$ Institute of Transportation, Fujian University of Technology, Fuzhou, Fujian, China \\ ${ }^{2}$ School of Modern Posts and Institute of Modern Posts, Nanjing University of Posts and Telecommunications, \\ Nanjing, Jiangsu, China \\ ${ }^{3}$ School of Transportation, Southeast University, Nanjing, Jiangsu, China \\ ${ }^{4}$ Department of Infrastructure Engineering, University of Melbourne, VIC, Australia
}

Correspondence should be addressed to Yue Zheng; zhengyue_seu@163.com

Received 13 December 2020; Revised 15 January 2021; Accepted 22 January 2021; Published 15 February 2021

Academic Editor: Tingsong Wang

Copyright (C) 2021 Liangpeng Gao et al. This is an open access article distributed under the Creative Commons Attribution License, which permits unrestricted use, distribution, and reproduction in any medium, provided the original work is properly cited.

\begin{abstract}
How to meet the daily demand for resident transport while limiting the transmission of infectious diseases is a problem of social responsibility of urban transport systems during major public health emergencies. Considering the novel coronavirus pneumonia epidemic (COVID-19), a bus timetable system based on the "if early, wait, and if late, leave soon" strategy is proposed. Based on public transport vehicle constraints in this system, the concept of reliability is introduced to evaluate public transport timetable systems, and a model based on an event tree is built to calculate the failure rate of urban bus timetables. Then, the public transport situation in Yixing city is used as an example to perform confirmatory analysis, and the fluctuations in the reliability of the bus timetable during the novel coronavirus pneumonia epidemic are discussed. The research results show that the method proposed in this paper can obtain the overall failure rate of urban bus timetable by traversing the calculation of each round-trip interval and achieve an accurate evaluation of the reliability of bus timetables. During the early, middle, and more recent stages of the COVID19 outbreak, the failure rate of bus timetables in Yixing city initially decreased and then increased. In the early stage of the outbreak, the failure rate of the Yixing bus timetable was 7.8142. However, the failure rate decreased to 4.3306 and 5.0160 in the middle and late stages of the epidemic, respectively. In other words, the failure rate of the public transport network in the middle and late stages decreased by $44.58 \%$ and $35.81 \%$, respectively, compared with that in the early stage. Thus, during major health emergencies, such as the novel coronavirus pneumonia outbreak, the reliability of the urban bus timetable system can be improved by at least $35 \%$, and cross-infection at bus stations can be prevented. The research results verify the feasibility and reliability of the implementation of bus timetabling strategies during major health emergencies.
\end{abstract}

\section{Introduction}

Major epidemics involving infectious diseases are serious public health events that can lead to the spread of infection sources across regions and borders and pose a threat to the safety of all human beings $[1,2]$. Taking effective measures to prevent, control, and eliminate the outbreak of major infectious diseases can not only reduce the degree of social harm caused and ensure the safety of individuals and property but also help maintain regional public order and promote harmonious social communication.

Since novel coronavirus pneumonia emerged in late December 2019, the COVID-19 epidemic has spread to many countries and regions around the world and has had a critical impact on individuals' daily life, security stability, and socioeconomic status. Through early June 2020, the COVID-19 disease, which has been reported in 188 countries/regions, had led to 420,000 deaths, and over 7.5 million 
cases were confirmed [3]. To delay and reduce the onset of peak infection effects, governments and public health authorities adopted a variety of social distancing interventions and policies to avoid overloading the health care system before a vaccine is available. Notably, a series of measures implemented by the Chinese government to block the spread of COVID-19 achieved an ideal health outcome [4].

From January 23 to 29, 2020, 31 provinces, cities, and regions in China successively launched the first level of national response to the major public health emergency. Specifically, prevention and control measures, such as complete travel bans, travel quarantines, and entry screening for incoming travellers, were mainly implemented [5]. In regard to urban transport systems, dual social responsibility, including providing basic traffic functions and blocking the spread of epidemics in the process of citizen travel, should be considered. Taking public transport as an example, in addition to regular epidemic prevention measures such as real-name boarding, passenger temperature monitoring, scattered seating, and the all-weather disinfection of vehicles, developing an operation mechanism to reduce the risk of crossinfection in bus travel and meet the rigid needs of passengers has become an urgent issue for scholars to consider.

Novel coronavirus pneumonia is mainly spread through droplets, contact, aerosols, faecal orifices, etc. [6]. Because of the rapid spreading and high morbidity characteristics of the virus, the urban public transport system needs to avoid mass gatherings of passengers in public places in the process of epidemic prevention. If virus-carrying passengers enter the public transport system, other people face a huge risk of infection, especially during rush-hour periods. An epidemic can quickly spread to every corner of a city due to the connectivity of bus lines among stations in different areas. If a bus timetabling system is used in conventional public transport, passengers can arrange their own itinerary and effectively reduce the waiting time at bus stations $[7,8]$. Thus, while meeting the rigid demands of residents for bus travel, the probability of contracting a virus while using public transport systems can be reduced. Moreover, the personal travel demand for leisure and social interaction has been greatly reduced, and the urban traffic situation has been significantly improved during the execution of social distancing policies. Therefore, it is possible for buses to travel smoothly through transportation networks, and the accuracy of the bus timetable is high.

We design a time control point method based on the "a vehicle would wait if it arrives early and leave quickly if it arrives late" scheme for a bus timetabling strategy to assess bus travel time performance. The aim of this study is to provide an evaluation method for analysing the characteristics of bus arrival with inbound and outbound information. By treating the upstream or downstream operation processes of a bus line as a single event, we construct an event tree model to explore the overall reliability of the public transport network. Finally, the public transport system in Yixing city, which is a county-level city with a population of one million in Southeast China, is studied to verify the feasibility and reliability of bus timetabling in the context of the COVID-19 epidemic.

\section{Literature Review}

As the fundamental dimension of passenger service, the reliability of urban public transport systems has been defined from several perspectives. According to the Transit Capacity and Quality of Service Manual [9], various factors that influence bus service reliability have been explored, such as the traffic conditions, road construction, maintenance quality, staff availability, schedule achievability, and passenger demand. Some achievements have been widely considered in bus system planning and design in many countries and regions, thereby providing a powerful scientific basis for the application of transit priority policies [10]. Specifically, many studies have focused on bus timetables. Therefore, this section focuses on the following three interrelated issues: timetable generation and optimization, vehicle and crew scheduling, and the operational strategy of bus system services.

2.1. Timetable Generation and Optimization. Timetable generation is one of the major tasks in the urban bus system [11]. A suitable bus timetable not only allows passengers to reach their destination quickly with as few bus routes as possible but also provides transfers with a short waiting time, which can greatly affect the service attractiveness of public transport systems [12]. The earliest research in this field can be traced to Newell and Potts's study of bus schedule maintenance, which identified the factors that influence the practical operation of bus systems [13]. Subsequently, an increasing number of scholars have explored the mechanisms of the associated phenomena, such as bus bunching [14-16], bus-specific holding [17-20], and dispatching control [21-24]. Although the focus of these studies varied, there is a certain correlation among each approach and the corresponding findings. For example, one purpose of dispatching control is to reduce the frequency of bus punching with a regularity-based or punctuality-based service strategy [25]. Compared with the regularity-based strategy, which minimizes the deviation in bus headways, the punctualitybased strategy focuses on the adherence to scheduled arrival times at stops, although some studies have found that passengers may ignore the exact arrival time when the schedule headway is less than $12 \mathrm{~min}$ [26-30]. Table 1 summarizes the recent research on the scheduling of bus timetable (including the train timetable) under the constraints of external factors.

Generally, there are two main research directions in the existing research: improving the performance of model algorithms and relaxing the modelling hypothesis to improve the representation of the objective world [45, 52-54]. The corresponding results of these studies include complex mathematical descriptions and rigorous derivation processes; therefore, the time costs of these methods can be high, but accurate calculation and evaluation results can be obtained.

2.2. Vehicle and Crew Scheduling. The vehicle and crew scheduling problem in bus systems is of practical importance because efficient schedules can reduce operational costs and 
TABLE 1: Recent research on the scheduling of bus timetable.

\begin{tabular}{|c|c|c|}
\hline Authors & Factors considered in the study & Notes \\
\hline Gkiotsalitis et al. [31-34] & $\begin{array}{l}\text { Rolling horizons bus fleet allocation, holding } \\
\text { control, and transit rescheduling strategies }\end{array}$ & $\begin{array}{l}\text { The purpose is to increase the coordination } \\
\text { among running buses, avoid vehicle bunching, } \\
\text { and obtain the accurate evaluation of bus } \\
\text { timetable }\end{array}$ \\
\hline $\begin{array}{l}\text { Salicru et al. [35]; Steiner and Irnich [36]; } \\
\text { Zhang et al. [37]; Ma et al. [38] }\end{array}$ & $\begin{array}{l}\text { Passenger travel demands extracted from } \\
\text { multisource traffic datasets }\end{array}$ & $\begin{array}{l}\text { Smarter computational methods were } \\
\text { provided to reduce operational costs and } \\
\text { improve the server level of bus timetable }\end{array}$ \\
\hline $\begin{array}{l}\text { Domschke [39]; Ceder et al. [40]; Eranki } \\
\text { [41]; Liu et al. [42]; Ibarra-Rojas et al. } \\
\text { [43-45] }\end{array}$ & $\begin{array}{l}\text { Bus line network, route choices of passengers, } \\
\text { waiting time at nodes, and the operational costs }\end{array}$ & $\begin{array}{l}\text { They developed a series of models to represent } \\
\text { the route choice behaviours of various } \\
\text { passengers and minimize the operational cost } \\
\text { of bus timetables }\end{array}$ \\
\hline $\begin{array}{l}\text { Wong et al. [46]; Shafahi and Khani [47]; } \\
\text { Kang et al. [48]; Guo et al. [49, 50]; Chu } \\
\text { et al. [12]; Abdolmaleki et al. [51] }\end{array}$ & $\begin{array}{l}\text { Trains' run times, station dwell times, } \\
\text { interchange waiting times of all passengers, } \\
\text { transfer redundant time, and the network } \\
\text { accessibility }\end{array}$ & $\begin{array}{l}\text { A series of nonlinear programming models } \\
\text { were provided to achieve the synchronize } \\
\text { timetables in the transit network and improve } \\
\text { the transfer efficiency of passengers }\end{array}$ \\
\hline
\end{tabular}

increase the capacity of transit services [55]. Related studies can be traced back to the 1940s when some scholars in developed countries introduced a series of approaches to maximize the scheduling profit $[56,57]$. Notably, in addition to minimizing the internal and external operating costs and synchronizing departure times, previous studies focused on the following aspects of timetable systems.

\subsubsection{Timetabling considering the Vehicle Scheduling} Efficiency. The research field of traditional bus scheduling covers all trips with fixed travel times and locations for the starting and ending points in a timetable [58]. Some studies mainly focused on minimizing the fleet size for a conventional bus service, which is a fixed timetable service with a single depot or single vehicle type [59-64]. Referring to the review of Ibrra-Rojas et al. [65], other studies were presented to solve multidepot vehicle scheduling problems [66, 67] and assess the robustness, network topology, and flexibility of different methods [44, 68]. Subsequent studies further improved the integration of vehicle timing and scheduling strategies [53, 69, 70], and other studies considered various practical factors, such as bus ridership, new vehicle types, skip-stop strategies, and functional planning periods, to make the mathematical model as similar to the actual daily bus travel situation as possible [36, 71-73].

2.2.2. Timetabling Integrated with Vehicle and Crew Scheduling. Early studies of vehicle scheduling and crew scheduling were formulated as network flow problems or sets of coverage or partitioning problems [74]. The integration of these two scheduling tasks can be regarded as a particular case of the vehicle scheduling problem that was first discussed by Gaffi and Nonato [75]. The following scholars applied various algorithms to relax the relevant constraints, such as duty flow, bus itinerary, overall operating cost, and multicommodity network constraints, to obtain satisfactory results [76-80]. These studies provided a functional framework for people to explore efficient timetables. For example, based on the achievements of Gintner et al. [81] and Steinzen et al. [82], Kliewer et al. [83] proposed a flexible timetable to reduce the operational costs and maintain the service quality by addressing the vehicle and driver scheduling problem with the possibility of shifting trips within defined time windows. Petersen et al. [84] proposed a model that integrated the bus network timetable and multidepot vehicle scheduling to minimize the usage costs of stocks and the transfer waiting times of passengers. The results indicated that flexibility in the bus timetables can reduce transfer waiting times by $20 \%$.

2.3. Operational Strategy for Bus System Services. As discussed above, bus holding, dispatching control, and vehicle and crew scheduling can be used as operational strategies to improve the service level of urban public transit. The Transit Capacity and Quality of Service Manual [9] also discusses some other measures, such as transit development plans, transit signal priority, park-and-ride schemes, and ticket pricing adjustment, that involve modern technical methods. However, not all technical measures can provide stable transit services because passenger behaviour influences the service quality of bus systems [85]. Therefore, scholars have considered a series of instances when exploring on-time performance, which has a strong relationship with bus timetables [86]. As a case of bus holding control, time control point rules that were applied in a previous study to evaluate network reliability were also used to solve dynamic engineering problems [23, 87-90]. For example, in recent studies, Van Oort et al. [91] introduced a method to increase the reliability of bus timetables by using the holding point strategy. The on-time arrival and departure characteristics of a bus station timetable were used to measure reliability. Dou et al. [92] developed a novel time control point strategy to obtain the optimal slack time scheme and applied an MINLP model to improve schedule adherence and reduce transfer waiting time. Wang et al. [93] proposed a joint strategy with bus holding and timetabling slack to optimize the locations of control points and formulate a bus timetable considering the uncertainty in the operational process. Other external 
factors, including the headway in a rolling horizon scheme, greenhouse gas emission policy, and bus line capacity, were also considered to integrate the vehicle procurement scheme and timetabling for urban transport [37, 94, 95].

In our previous work, we proposed a slack arrival strategy in which transit vehicles are allowed to reach checkpoints somewhat later than the scheduled departure time and delayed vehicles must leave the checkpoints immediately after serving the boarding and alighting passengers [96]. In this paper, we extended this strategy of time control points to the environment of the COVID-19 epidemic and proposed a reliability evaluation model to quickly acquire and identify which intervals in the bus network would have large arrival delays or transfer synchronization problems in the operational process. The contributions of this paper are listed as follows:

(1) A system analysis framework for estimating the overall reliability of the time control point strategy is provided considering the spread of the COVID-19 epidemic in the bus network

(2) A first-order reliability method and Ditlevsen's bounds theory are integrated to simplify the reliability calculation and improve the operating speed of the bus network

(3) The event tree approach is applied to analyse the real case of Yixing city during the epidemic considering vehicle arrival delays for multiple bus lines

The remainder of this paper is structured as follows. Section 3 presents the methodology for calculating the reliability of the bus network, and a first-order reliability method and Ditlevsen's bounds theory are integrated. Section 4 introduces the case study and provides an analysis of the results. Section 5 presents a summary of the study and gives suggestions for future studies.

\section{Methodology}

3.1. Problem Description. Generally, the public recognition of major public health emergencies is a gradual process, especially for the transmission route, transmission mechanism, vaccine research, and development cycle, which are difficult to constrain; as a result, the requirements for the formulation of epidemic prevention measures are extensive. In this outbreak of COVID-19, the Chinese government took a series of active social distancing measures to prevent the spread of the virus and achieved good results. These measures not only reduced human contact but also significantly improved urban traffic conditions, which made it possible for bus stations to adopt operational strategies based on bus timetabling.

Under this timetable system, buses that arrive earlier than the planned arrival time need to wait until the departure time is reached based on the time control points established for the bus station, and buses that arrive late leave as soon as possible on the basis of ensuring the service of passengers. The arrival time at each bus station is often accurate to the minute. From the perspective of reliability, if a bus arrives at a station before the expected time, it is considered an on-time arrival, and the bus service is in a "reliable" state. Otherwise, if a bus fails to meet the time point condition, it will be considered late. To determine the overall reliability rate of the system, we must first calculate the reliability of each station interval and then estimate the overall reliability of the bus route. Finally, the event tree analysis method is applied to determine the reliability of the whole public transport network. In the following subsection, we introduce a three-stage reliability calculation framework for the bus timetabling strategy.

3.2. Stage 1: Reliability of the Arrival Interval. According to the previous description, when a bus starts from an upstream station and reaches a downstream station before the specified time point, the bus is considered "reliable." Therefore, under normal conditions, each interval between two adjacent stations has a planned arrival time $\operatorname{Tr}_{i j}$ and an actual arrival time $A r_{i j}$. The performance of the punctuality index $G r_{i j}$ refers to the difference between these two arrival time points and can be computed from the following formula:

$$
G r_{i j}=T r_{i j}-A r_{i j} \forall, \quad i \in(1, n-1), j \in(2, n), i \neq j,
$$

where $i$ and $j$ refer to the corresponding values at two adjacent stations along the same route.

Based on the punctuality index $G r_{i j}$, we can determine whether vehicles can reach the downstream station according to the bus timetable. If the value of this index is larger than zero, i.e., the vehicle arrives at the downstream station on time, the timetable status is reliable. Otherwise, the time requirements are not met, and the timetable is invalid. We assume that during the epidemic period, passengers can obtain the bus timetable through various information channels in real time. Additionally, to shorten the waiting time of passengers at a bus station, which may lead to the spread of coronavirus, bus companies will adjust the timetables of subsequent bus stations according to the real-time arrival of each bus, and these changes are announced in real time. In the actual operation of a bus timetable, a vehicle often shuttles forward and backward between bus stations, so the travel time difference in each interval can be regarded as a set. By using a mathematical method, we can quantify the reliability and failure characteristics of the timetable for each interval. Therefore, when the bus timetable is dynamically updated, we can assume that the planned time point and actual time point of bus arrival both satisfy the normal distribution, e.g., $\operatorname{Tr}_{i j} \sim\left(\mu_{T i j}, \sigma_{T i j}^{2}\right)$ and $A r_{i j} \sim\left(\mu_{A i j}, \sigma_{A i j}^{2}\right)$. Therefore, the punctuality index $G r_{i j}$ also satisfies the normal distribution, e.g., $G r_{i j} \sim\left(\mu_{T i j}-\mu_{A i j}\right.$, $\left.\sigma_{T i j}^{2}+\sigma_{A i j}^{2}\right)$. We can obtain the following result after the standard normal transformation:

$$
\frac{G r_{i j}-\left(\mu_{T i j}-\mu_{A i j}\right)}{\sqrt{\sigma_{T i j}^{2}+\sigma_{A i j}^{2}}} \sim(0,1)
$$

By setting $W=G r_{i j}-\left(\mu_{T i j}-\mu_{A i j}\right) / \sqrt{\sigma_{T i j}^{2}+\sigma_{A i j}^{2}}$, we can obtain $G r_{i j}=W \cdot \sqrt{\sigma_{T i j}^{2}+\sigma_{A i j}^{2}}+\left(\mu_{T i j}-\mu_{A i j}\right)$. If the timetable of a bus line fails, e.g., $G r_{i j}<0, W<-\left(\mu_{T i j}-\mu_{A i j}\right) /$ 
$\sqrt{\sigma_{T i j}^{2}+\sigma_{A i j}^{2}}$. Therefore, the reliability index $r_{i j}$ of each interval can be calculated using the following formula:

$$
r_{i j}=\frac{\mu_{T i j}-\mu_{A i j}}{\sqrt{\sigma_{T i j}^{2}+\sigma_{A i j}^{2}}} .
$$

According to the first-order reliability method [97, 98], the probability of an arrival delay between two adjacent stations due to various factors can be determined by the following formula:

$$
P\left(G r_{i j}<0\right)=P\left(W<\frac{-\left(\mu_{T i j}-\mu_{A i j}\right)}{\sqrt{\sigma_{T i j}^{2}+\sigma_{A i j}^{2}}}\right),
$$

where $P\left(G r_{i j}<0\right)$ refers to the failure probability at the downstream station and the corresponding reliability for a given interval can be calculated according to $1-P\left(G r_{i j}<0\right)$.

3.3. Stage 2: Reliability of Bus Route. In an actual transit network, a bus route contains several subroutes and often covers multiple intervals. Considering the operational mechanism of a bus timetable, the reliability of a bus route depends on whether the bus can reach stations on time. If the bus is delayed in any interval, it may lead to the failure to reach the downstream bus station according to the timetable; as a result, the subsequent timetable for the bus trip will be in a "nonreliable" state. Therefore, when assessing the overall reliability of the timetable of a bus line, the operational state of the timetable should be considered in each interval of the bus route.

Figure 1 is the operation status diagram for a bus timetable. The solid line in the figure shows that the travel time difference between two adjacent stations is greater than 0 or equal to 0 , e.g., $G r_{i j} \geq 0$, and the dotted line represents a value less than 0, e.g., $G r_{i j}<0$. For example, in scenario (a) in Figure 1, the bus is delayed in interval 1, and the subsequent timetable of the route is invalid. The failure probability can be obtained from the following formula:

$$
P_{f}=P\left(F_{1}\right) \text {. }
$$

In scenario (b) in Figure 1, part of the arrival delay shifts from interval 1 to interval 2. According to set theory, the failure probability for this situation can be calculated by

$$
P_{f}=P\left(F_{2} \cap S_{1}\right) \text {. }
$$

Similarly, as shown in scenario (c) in Figure 1, when an arrival delay occurs in interval 3 , the corresponding probability is

$$
P_{f}=P\left(F_{3} \cap S_{2} \cap S_{1}\right)
$$

Analogously, when the timetable fails in interval $n$, the formula for the probability is as follows:

$$
P_{f}=P\left(F_{n} \cap S_{n-1} \cap S_{n-2} \cdots \cap S_{2} \cap S_{1}\right) .
$$

According to set theory, the failure probability of a timetable for a bus route is as follows:

$$
\begin{aligned}
P_{f}= & \left.P\left(F_{1}\right)\right)+P\left(F_{2} \cap S_{1}\right)+P\left(F_{3} \cap S_{2} \cap S_{1}\right) \cdots \\
& +P\left(F_{n} \cap S_{n-1} \cap S_{n-2} \cdots \cap S_{2} \cap S_{1}\right) .
\end{aligned}
$$

If the timetable state of each interval on the bus route is regarded as a set $R$, two subsets can be obtained accordingly: the reliable subset $S$ and failure subset $F$. The arrivals delays that occur in intervals 2 and 3 in scenarios (b) and (c) in Figure 1 can be expressed as follows:

$$
\begin{aligned}
P_{f} & =P\left(F_{2} \cap S_{1}\right)=P\left\{F_{2} \cap\left(R-F_{1}\right)\right\} \\
& =P\left(F_{2} \cap R\right)-P\left(F_{2} \cap F_{1}\right) \\
& =P\left(F_{2}\right)-P\left(F_{2} \cap F_{1}\right), \\
P_{f} & =P\left\{F_{3} \cap\left(R-F_{2}\right) \cap\left(R-F_{1}\right)\right\} \\
& =P\left\{\left(F_{3}-F_{2} \cap F_{3}\right) \cap\left(R-F_{1}\right)\right\} \\
& =P\left(F_{3}\right)-P\left(F_{2} \cap F_{3}\right)-P\left(F_{3} \cap F_{1}\right)+P\left(F_{3} \cap F_{2} \cap F_{1}\right) .
\end{aligned}
$$

Moreover, the formula for calculating the failure probability of a bus route can be deduced as follows:

$$
\begin{aligned}
P_{f} & =P\left(F_{1}\right)+P\left(F_{2}\right)-P\left(F_{2} \cap F_{1}\right)+P\left(F_{3}\right)-P\left(F_{3} \cap F\right)_{1}-P\left(F_{3} \cap F_{2}\right)+P\left(F_{3} \cap F_{2} \cap F_{1}\right)+\cdots \\
& =\sum_{g=1}^{n} P\left(F_{g}\right)-\sum \sum_{g<h}^{n} P\left(F_{g} \cap F_{h}\right)+\sum \sum \sum_{g<h<k}^{n} P\left(F_{g} \cap F_{h} \cap F_{k}\right)-\cdots
\end{aligned}
$$

Formula (11) shows that the number of calculations required to determine the failure probability for the timetable of a bus route will increase with the number of intervals, which is not conducive to the rapid evaluation of reliability based on the time control point strategy. Therefore, this paper considers using the upper and lower boundary values of the failure probability to simplify the calculation process and improve the computational efficiency. According to the narrow bound theory, the following relationship exists for any adjacent stations along a bus route:

$$
P\left(S_{n-1} \cap S_{n-2} \cdots \cap S_{1}\right) \leq P\left(S_{n}\right)=P\left(1-F_{n}\right) .
$$

By applying formulas (11) and (12), we can obtain 


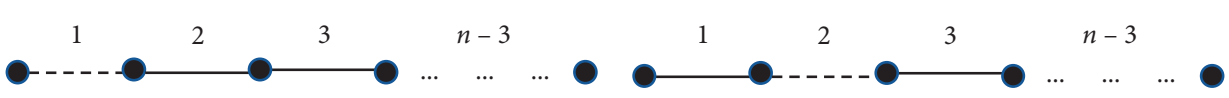

(a)

(b)

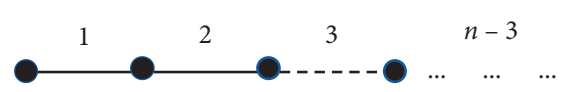

(c)

Figure 1: Operational state diagram for a bus timetable.

$$
P_{f} \leq \sum_{g=1}^{n} P\left(F_{g}\right)-\sum_{h=2}^{n} \max _{h<k}\left\{P\left(F_{h} \cap F_{k}\right)\right\} .
$$

In addition, for any interval, the following relationships can be established:

$$
\begin{gathered}
P\left(S_{n-1} \cap S_{n-2} \cdots \cap S_{1}\right) \geq 1-\left\{P\left(F_{1}\right)+P\left(F_{2}\right)+\cdots+P\left(F_{n-1}\right)\right\} \\
P\left(F_{1} \cap S_{n-1} \cap S_{n-2} \cdots \cap S_{1}\right) \geq P\left(F_{1}\right)-\sum_{g=1}^{n-1} P\left(F_{1} \cap F_{g}\right) \\
P_{f} \geq P\left(F_{1}\right)+\sum_{g=2}^{n} \max \left\{P\left(F_{g}\right)-\sum_{h=1}^{g-1} P\left(F_{g} \cap F_{h}\right), 0\right\} .
\end{gathered}
$$

According to the Ditlevsen bounds theory, the upper and lower limits of the failure probability of timetables for bus routes can be obtained as follows:

$$
\begin{aligned}
& P_{\text {upper }}=\sum_{g=1}^{n} P\left(F_{g}\right)-\sum_{h=2}^{n} \max _{h<k}\left\{P\left(F_{h} \cap F_{k}\right)\right\}, \\
& P_{\text {lower }}=P\left(F_{1}\right)+\sum_{g=2}^{n} \max \left\{P\left(F_{g}\right)-\sum_{h=1}^{g-1} P\left(F_{g} \cap F_{h}\right), 0\right\},
\end{aligned}
$$

where $P_{\text {upper }}$ refers to the upper limit of the failure probability and $P_{\text {lower }}$ refers to the lower limit of the failure probability. To facilitate the calculation, the failure rate is taken as the mean value of the upper and lower limits:

$$
P_{f} \approx \frac{1}{2}\left(P_{\text {upper }}+P_{\text {lower }}\right) \text {. }
$$

3.4. Stage 3: Reliability of a Transit Network. A transit network is composed of bus lines and stations. Bus stations can be regarded as nodes in a network diagram that are connected by the route intervals mentioned above. The overall structure of an urban transit network influences the convenience and timetable reliability of residents' bus trips and directly affects the operation efficiency of the public transport system. In daily life, residents often expect to be able to transfer through adjacent bus stations or the same station in a short time to conveniently complete their bus trips. Therefore, we assume that if residents transfer more than two times in a single bus trip, they will consider the travel experience poor. Since the novel coronavirus pneumonia outbreak, people have focused on reasonable travel planning and become aware of the risk of transit transfer.

When analysing the reliability of a transit network, under the background of an epidemic situation, it is assumed that the daily bus trips of residents will not include more than 2 transfers. If the residents that travel via bus during the epidemic period are regarded as independent events without mutual interference, the network reliability can be calculated by analysing the travel structure chart with event tree traversal based on the above research results.

Figure 2 shows the event tree structure for passenger bus trips with no more than 2 transfers. Event tree analysis is an analysis method in which a tree view is applied to obtain the entire occurrence process for a series of events. The tree starts from the event occurrence, and the various development directions can be individually assessed. Each development direction is completely independent and has a corresponding event consequence [99]. For bus trips in the context of the COVID-19 epidemic, each branch in the event tree structure diagram represents a bus route that residents may choose when travelling. The output results of the event tree represent various bus routes. The occurrence probability of each failure mode $\left(\mathrm{FM}_{m}\right)$ in Figure 2 can be obtained by traversing all output results, and the bottleneck areas of the transit network can be identified. Among all the failure modes, those with the highest failure rates are the most likely to fail in the timetable. Additionally, the failure conditions of each bus route are independent, and the failure rate of each output in the event tree must satisfy the following relationship:

$$
P\left(\mathrm{FM}_{r}\right)=P\left(F_{o} \cap F_{p} \cap F_{q}\right)
$$




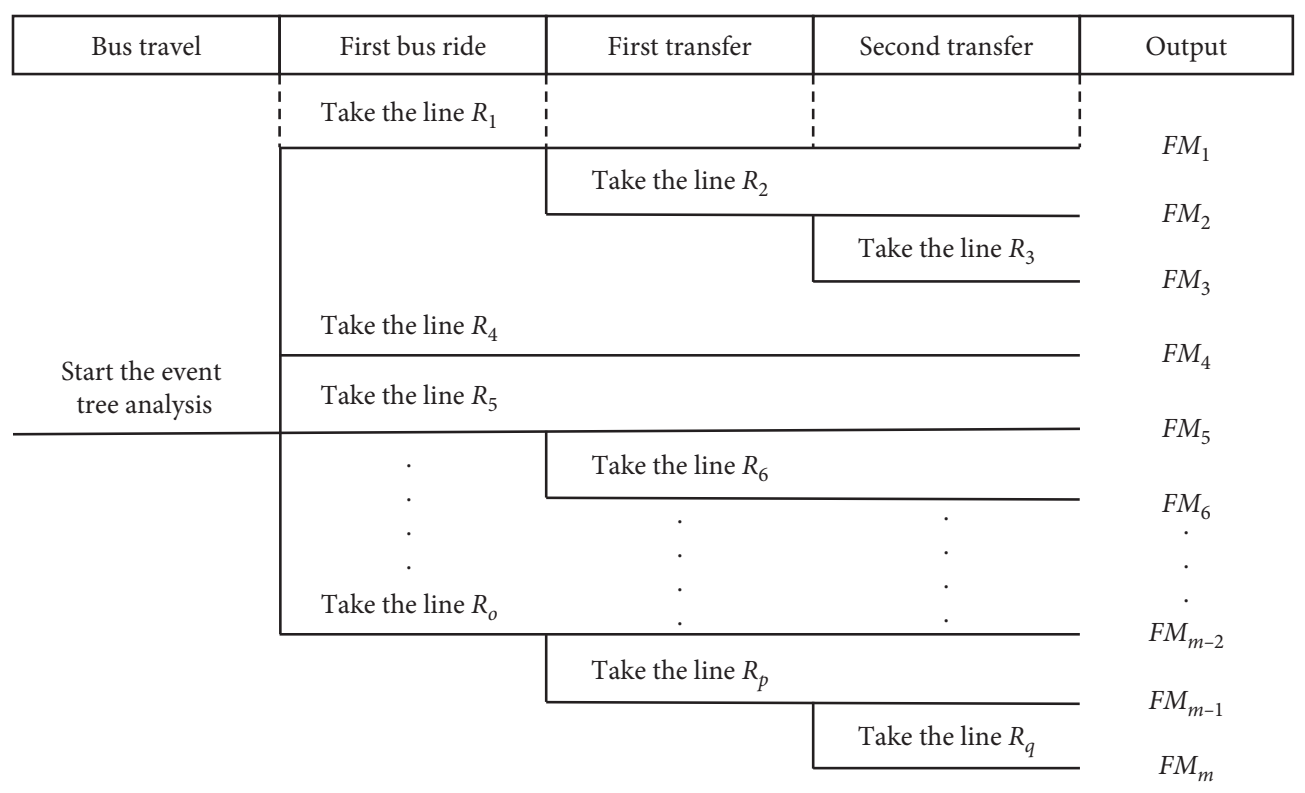

FIgURE 2: The structure of the event tree based on bus trips.

where $\mathrm{FM}_{r}$ refers to a failed bus timetable and the overall failure rate of the transit network should be the maximum value for all event results. The higher this value is, the lower the reliability of the timetable in the transit network is; conversely, the smaller the value is, the more reliable the timetable is.

\section{Results}

4.1. Case Study. To test the feasibility and effectiveness of the proposed method, Yixing city is selected as a case study. Yixing is a county-level city managed by Wuxi city, which is located in the southwestern part of Jiangsu province. The city is situated in the centre of the Shanghai-Nanjing-Hangzhou triangle (three large cities in Southeast China) and includes 5 blocks and 13 towns. At present, there are 51 bus lines in Yixing city, with a total length of $246.4 \mathrm{~km}$, an average daily departure frequency of 5400, and an average daily traffic volume of 166,000 persons. The overall density of the public transport network in the city is $1.48 \mathrm{~km} / \mathrm{km}^{2}$, and the sharing rate of bus travel in the urban traffic structure reaches $26 \%$. Urban public transport has become an important mode of daily travel for Yixing citizens. Figure 3 shows the location of Yixing city.

Yixing Public Transport Co., Ltd., is the only urban public transport enterprise in Yixing city. Since the outbreak of the COVID-19 epidemic in January 2020, rapid and effective adjustments to the bus operation lines in Yixing city have been made. To smooth the operation of the urban public transport system during the Spring Festival and try to reduce the waste of transit capacity resources, the number of bus lines in Yixing city was adjusted on January 20th. Then, the number of bus trips was reduced by two-thirds on January 27th (according to the original plan, one shift was implemented, and then a pause occurred for two shifts) to promote epidemic prevention and control while meeting the

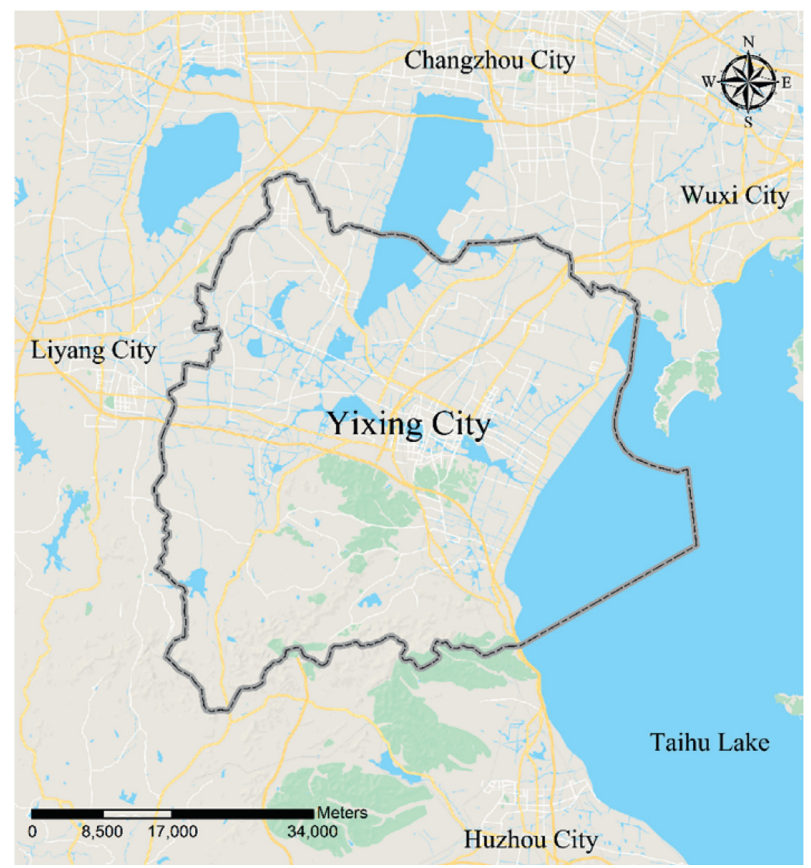

Figure 3: The location map of Yixing city.

basic bus travel needs of residents. When the novel coronavirus pneumonia epidemic entered the severe stage on February 8th, the bus operation schedule in Yixing was adjusted again, and the departure frequency of the 26 main bus routes was further reduced to 2 per day. In the context of the COVID-19 epidemic, Yixing city adopted a timetabling strategy with time control points, and this strategy was implemented through March 25th. Thus, in the process of epidemic prevention and control, Yixing applied a public transport operation control scheme that gradually increased in strictness according to the actual situation, and a bus timetable similar to a time control point strategy was used 


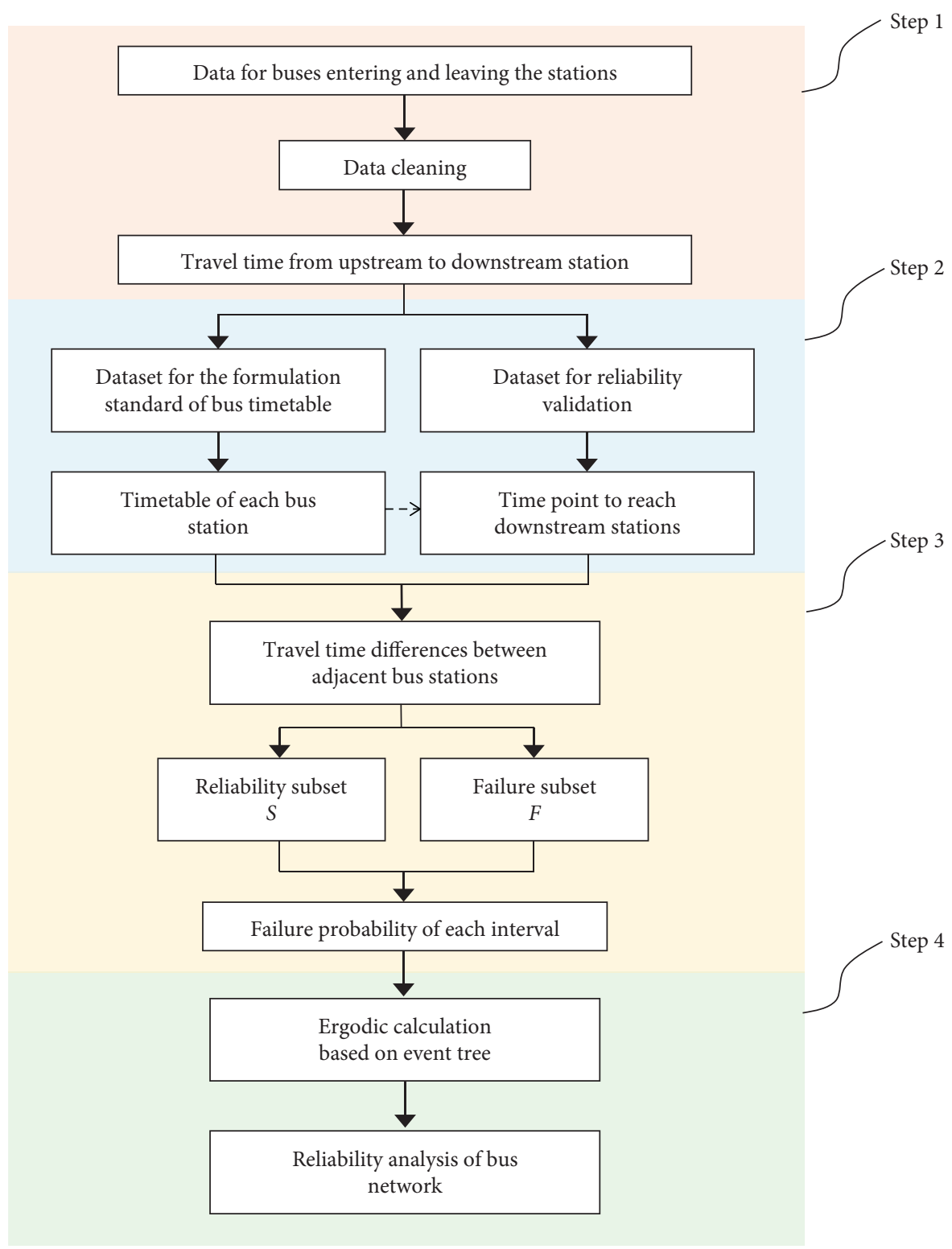

FIGURE 4: Flowchart for the reliability assessment of the bus timetable.

for up to two months. Therefore, the analysis data from Yixing city collected between December 1, 2019, and March 31,2020 , are used to evaluate the reliability of the bus timetable system during a major health emergency.

4.2. Data Analysis and Results. Figure 4 shows the flowchart of our study, which uses Yixing data to evaluate the reliability of the bus timetable. As mentioned above, the reliability of the overall system depends on the total number of bus routes and the on-time performance in each interval. The specific process steps are as follows.

(i) Step 1. We cleaned the data for buses entering and leaving the stations and eliminated missing and abnormal bus records.

(ii) Step 2. We divided the extracted punctuality index of the interval travel time into two parts. The first part was the interval travel time in December 2019, which was used to formulate the timetables of bus routes. The second part was the interval travel time between January and March 2020, which was used to verify the reliability of bus timetables during a major public health emergency.

(iii) Step 3. According to the bus timetables, we extracted the planned travel times from upstream to downstream stations. Then, we calculated the travel time differences based on the actual arrival time and made appropriate comparisons. If the travel time difference was larger than 0 , we considered the bus timetable "reliable"; otherwise, the bus timetable was "nonreliable."

(iv) Step 4. We calculated the failure probability of each bus route and constructed the event tree for resident bus travel during the epidemic period. The overall 


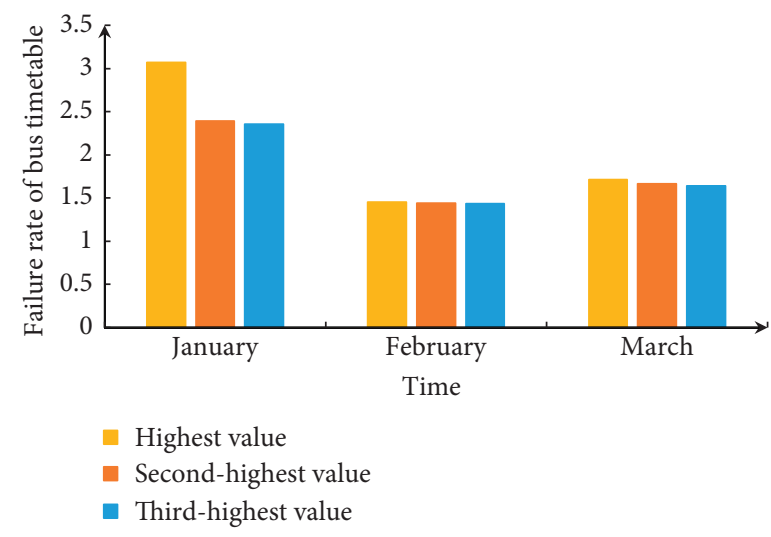

Figure 5: Peak diagram of timetable failure rate.

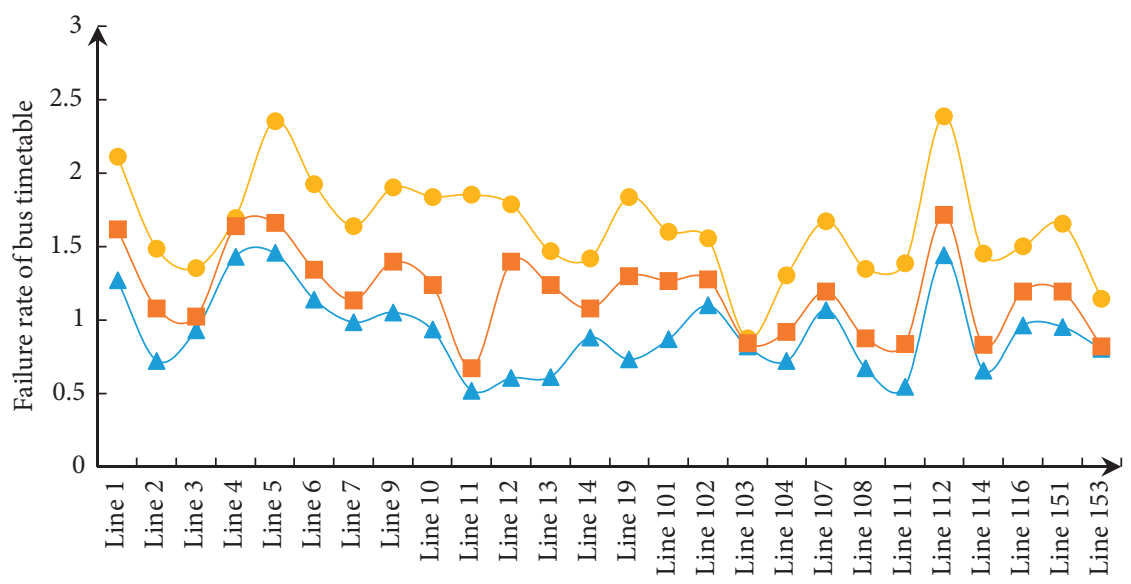

Bus lines in continuous operation from January to march

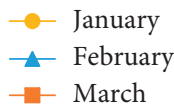

FIgURE 6: Timetable failure rate of each bus line under epidemic.

reliability of the timetable for the urban transit network was calculated and analysed.

To verify the reliability of the bus timetable, the $75 \%$ quantiles of the bus travel time in each interval before the outbreak of the epidemic were used as part of the failure criterion in Step 2. If a bus arrives at the downstream station prior to a given time, the timetable is considered reliable. The bus timetable is set in minutes, and the travel times are rounded up to the nearest minute for ease of understanding by residents. Moreover, this study eliminates the data for bus routes used less than 30 times in the same month to avoid the statistical bias caused by data collection.

Figure 5 shows the highest, second-highest, and thirdhighest failure rates for bus timetables in Yixing from January to March. Under the time control point strategy, the failure rate in January was significantly higher than that in February or March because of the arrival of the Spring Festival and the outbreak of the COVID-19 epidemic. Additionally, the urban traffic conditions significantly improved in late January, and the reliability of the bus timetabling strategy also improved; this improvement lasted until late March.

Figure 6 shows the bus lines that operated steadily in Yixing during the epidemic period and the corresponding failure rate under the time control point strategy. Notably, in the actual operation process, the failure rate of various lines displays certain volatility, but overall, the failure rate in January is higher than that in February or March. Moreover, the failure rate of most bus lines in February was lower than that in March because the residents of Yixing gradually returned to work and school during the later stage of the COVID-19 epidemic; in addition, the bus lines in Yixing resumed normal operation based on the original schedule. In the epidemic prevention process, the reliability of bus timetables gradually improved due to the lack of urban traffic. Furthermore, we used event tree analysis to traverse the timetable failure rate in Yixing city from January to March and found that the failure rate of the transit network was 7.8142 in the early stage of the COVID-19 


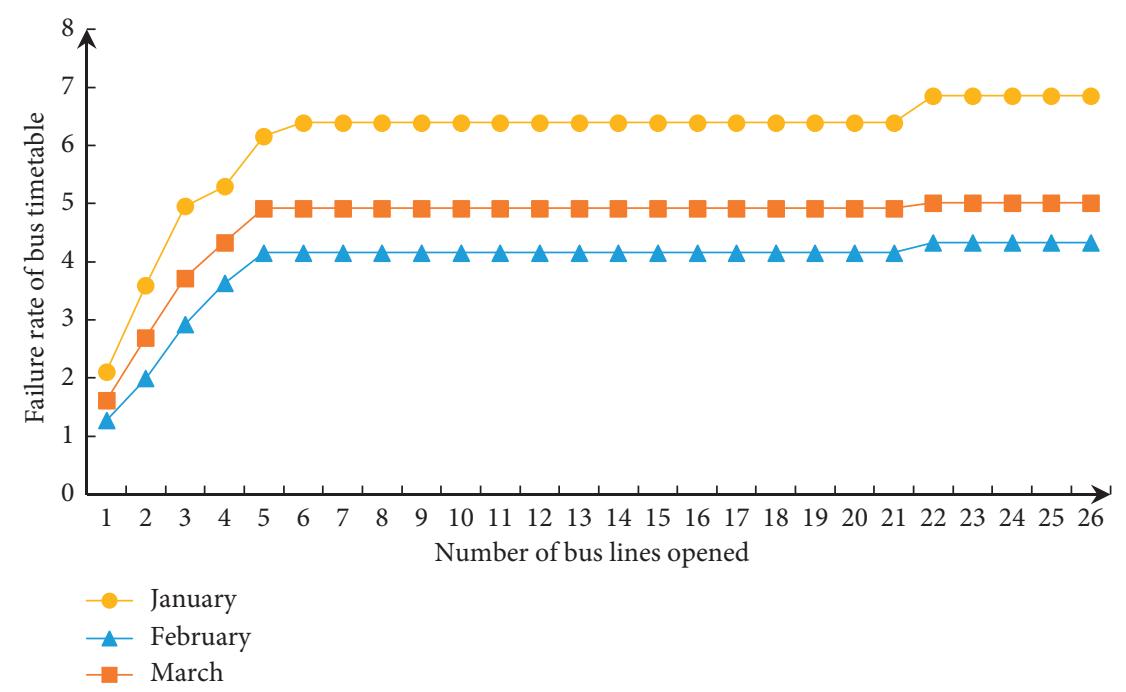

Figure 7: Timetable failure rate for each bus line during the pandemic.

epidemic outbreak and 4.3306 and 5.0160 in the middle and late stages, respectively. The failure rates of the timetables in the middle and late stages of the epidemic decreased by $44.58 \%$ and $35.81 \%$ compared with that in the early stage, respectively (Figure 7).

In regard to the evolution of the failure rate, we can see that as the number of bus lines opened increases from 1 to 26, the COVID-19 pandemic has a gradually increasing negative impact on the reliability of bus timetables. However, from the perspective of increment, the failure rate of bus timetables increases and tends to be stable when the bus network has a growing scale of operation. This shows that the negative influence of the bus line number follows the principle of diminishing marginal utility to affect the reliability of the timetable. If the number of bus lines is small, the failure rate of the bus timetable shows a significant increasing trend, and when the number of timetables exceeds 6 , the reliability enters a step-by-step decline stage. Thus, when planning the bus timetable, policy makers should pay attention to the relationship between the difficulty of pandemic transmission and the number of bus lines to avoid a high risk of epidemic spread caused by insufficient bus capacity or too many bus lines.

\section{Conclusions}

In this paper, we proposed a reliability analysis framework to evaluate the bus timetabling strategy for a transit network during the COVID-19 epidemic period. By integrating the first-order reliability method and Ditlevsen's bounds theory, a detailed formula derivation process and calculation steps were included in the analysis framework. Three scenarios, e.g., the early, middle, and late stages of the epidemic outbreak, were considered to verify the validity of the model. The failure rate of the bus timetabling strategy, an important indicator used to reflect the operational reliability of the transit network, can be obtained quickly from statistical computations involving the model output.
The reliability analysis framework developed here can be used to determine how to apply data for buses entering and leaving stations to dynamically determine the punctuality characteristics of the time control point strategy. Event tree analysis was used in this study to compute the timetable failure rate considering the transfer and waiting times of residents at bus stations. Each bus trip in the model was regarded as an independence event, and the events did not affect each other in the probability calculation. Because of the bottleneck effect, the highest value of failure probability for all events was the final rate for the entire transit network. The model calibration results illustrate that, in the context of major health emergencies, it is not only feasible but also important to implement the time control point strategy of "if a bus arrives early, it must wait, and if a bus arrives late, it should leave as quickly as possible." According to the case study of Yixing city, the failure rate of the bus timetable initially decreased and then increased based on the analysis framework. Compared with that in the early stage of the epidemic, the failure rate in the middle and late stages decreased by $44.58 \%$ and $35.81 \%$, respectively. This finding suggests that the reliability of the timetabling strategy increased by at least $35 \%$ during the epidemic period.

Overall, the notable innovation of this paper is to provide a refined reliability measurement method based on the failure rate of bus timetables. The calculation framework is a convenient and easily applicable evaluation model which does not consider the topology of bus line network. Compared with the previous studies, the final result can be ideally obtained by traversing the calculation of the failure probability of each round-trip interval. The decision makers could quickly find out the weak point of bus timetable in the network and work out the corresponding solutions. However, because the evaluation process does not consider the influence of the network topology fully, the results may be insufficient in some situation, such as under the environment of extreme congestion. Further research should explore a refined reliability measurement 
method and combine such a method with real-time bus travel data to build a dynamic evaluation model for considering the special environments. And the functional requirements of synchronous multistation evaluation and reliable early warnings for various bus scheduling operation schemes should also be in view in the further reliability framework of bus timetable.

\section{Data Availability}

The data used to support the findings of this study are available from the corresponding author upon request.

\section{Conflicts of Interest}

The authors declare that they have no conflicts of interest regarding the publication of this paper.

\section{Acknowledgments}

The financial support from the National Key R\&D Program of China (no. 2018YFE0120100), the Natural Science Foundation of Fujian Province (2020J05194), the Education and Scientific Research Foundation of Fujian Finance Department (GY-Z21001), and the Technology Program of Fujian University of Technology (GY-Z19094 and GYZ17155) is gratefully acknowledged.

\section{References}

[1] R. Anderson and R. May, Infectious Diseases of Humans: Dynamics and Control, Oxford University Press, Oxford, England, 1995.

[2] J. Mossong, N. Hens, P. Beutels et al., "Social contacts and mixing patterns relevant to the spread of infectious diseases," PLoS Medicine, vol. 5, no. 3, pp. 381-391, 2008.

[3] "Johns Hopkins University, Coronavirus Resource Center," http://coronvirus.jhu.edu/(2020-06-01).

[4] M. U. G. KraemerC.-H. Yang et al., "The effect of human mobility and control measures on the COVID-19 epidemic in China," Science, vol. 368, no. 6490, pp. 493-497, 2020.

[5] A. AnzaiT. Kobayashi et al., "Assessing the impact of reduced travel on exportation dynamics of novel coronavirus infection (COVID-19)," Journal of Clinical Medicine, vol. 9, no. 2, p. $601,2020$.

[6] G.-Q. SunS.-F. Wang et al., "Transmission dynamics of COVID-19 in Wuhan, China: effects of lockdown and medical resources," Nonlinear Dynamics, vol. 101, no. 3, p. 1981, 2020.

[7] I. Kaddoura, B. Kickhöfer, and A. Neumann, A. Tirachini, "Agent-based optimisation of public transport supply and pricing: impacts of activity scheduling decisions and simulation randomness," Transportation, vol. 42, no. 6, pp. 1039-1061, 2015.

[8] G. K. D. Saharidis, C. Dimitropoulos, and E. Skordilis, "Minimizing waiting times at transitional nodes for public bus transportation in Greece," Operational Research, vol. 14, no. 3, pp. 341-359, 2013.

[9] Trasportation Research Board, Transit Capacity and Quality of Service Manual, Third Edition, The National Academies Press, Washington, DC, USA, 2013.

[10] National Academies of Sciences, "Engineering, and medicine". Using Archived AVL-APC Data to Improve Transit
Performance and Management. The National Academies Press, Washington, DC, USA, 2006.

[11] A. Ceder, Public Transit Planning and Opertaion: Theory, Modeling and Practice. Routledge \& CRC Press, Boca Raton, FL, USA, 2007.

[12] J. C. Ho, K. Korsesthakarn, and Y.-T. Hsu, H.-Y. Wu, "Models and a solution algorithm for planning transfer synchronization of bus timetables," Transportation Research Part E: Logistics and Transportation Review, vol. 131, pp. 247-266, 2019.

[13] G. F. Newell and R. B. Potts, "Maintaining a bus schedule," in Proceedings of the 2nd Australian Road Research Board Conference, Perth, Australia, August 1964.

[14] D. Helbing and B. Tilch, "Generalized force model of traffic dynamics," Physical Review E, vol. 58, no. 1, pp. 133-138, 1998.

[15] M. D. Hickman, "An analytic stochastic model for the transit vehicle holding problem," Transportation Science, vol. 35, no. 3, pp. 215-237, 2001.

[16] E. E. Osuna and G. F. Newell, "Control strategies for an idealized public transportation system," Transportation Science, vol. 6, no. 1, pp. 52-72, 1972.

[17] X. J. Eberlein, N. H. M. Wilson, and D. Bernstein, "Modeling real-time control strategies in public transit operations," Lecture Notes in Economic and Mathematical Systems, Springer, Berlin, Germany, 1999.

[18] L. Fu and X. Yang, "Design and implementation of busholding control strategies with real-time information," Journal of the Transportation Research Board, vol. 1791, pp. 6-12, 2001.

[19] P. Marguier, Bus route performance evaluation under stochastic conditions, Ph.D. thesis, Massachusetts Institute of Technology, Combridge, MA, USA, 1985.

[20] G. F. Newell, "Control of pairing of vehicles on a public transportation route, two vehicles, one control point," Transportation Science, vol. 8, no. 3, pp. 248-264, 1974.

[21] A. Adamski and A. Turnau, "Simulation support tool for realtime dispatching control in public transport," Transportation Research Part A: Policy and Practice, vol. 32, no. 2, pp. 73-87, 1998.

[22] A. Kinoshita, "On controlling randomness in transit operations," Transportation Science, vol. 8, no. 2, pp. 102-116, 1974.

[23] J. G. StrathmanK. J. Dueker et al., "Automated bus dispatching, operations control, and service reliability: baseline analysis," Transportation Research Record: Journal of the Transportation Research Board, vol. 1666, no. 1, pp. 28-36, 1999.

[24] A. Sun and M. Hickman, "The real-time stop-skipping problem," Journal of Intelligent Transportation Systems, vol. 9, no. 2, pp. 91-109, 2005

[25] M. Trompet, X. Liu, and D. Graham, "Development of Key performance indicator to compare regularity of service between urban bus operators," Transportation Research Record: Journal of the Transportation Research Board, vol. 1791, pp. 6-12, 2001.

[26] J. J. Bartholdi and D. D. Eisenstein, "A self-coördinating bus route to resist bus bunching," Transportation Research Part B: Methodological, vol. 46, no. 4, pp. 481-491, 2012.

[27] X. Chen, L. Yu, and Y. Zhang, J. Guo, "Analyzing urban bus service reliability at the stop, route, and network levels," Transportation Research Part A: Policy and Practice, vol. 43, no. 8, pp. 722-734, 2009.

[28] M. Dessouky, R. Hall, and L. Zhang, A. Singh, "Real-time control of buses for schedule coordination at a terminal," 
Transportation Research Part A: Policy and Practice, vol. 37, no. 2, pp. 145-164, 2003.

[29] X. J. Eberlein, N. H. M. Wilson, and D. Bernstein, "The holding problem with real-time information available," Transportation Science, vol. 35, no. 1, pp. 1-18, 2001.

[30] J. G. Gerhart, T. J. Kimpel, and K. J. Dueker, R. L. Gerhart, S. Callas, "Evaluation of transit operations: data applications of tri-met's automated," Transportation, vol. 29, no. 3, pp. 321-345, 2002.

[31] K. Gkiotsalitis and A. Stathopoulos, "Demand-responsive public transportation Re-scheduling for adjusting to the joint leisure activity demand," International Journal of Transportation Science and Technology, vol. 5, no. 2, pp. 68-82, 2016.

[32] K. Gkiotsalitis and E. C. van Berkum, "An exact method for the bus dispatching problem in rolling horizons," Transportation Research Part C: Emerging Technologies, vol. 110, pp. 143-165, 2020.

[33] K. Gkiotsalitis and O. Cats, "Reliable frequency determination: incorporating information on service uncertainty when setting dispatching headways," Transportation Research Part C: Emerging Technologies, vol. 88, pp. 187-207, 2018.

[34] K. Gkiotsalitis, Z. Wu, and O. Cats, "A cost-minimization model for bus fleet allocation featuring the tactical generation of short-turning and interlining options," Transportation Research Part C: Emerging Technologies, vol. 98, pp. 14-36, 2019.

[35] M. Salicrú, C. Fleurent, and J. M. Armengol, “Timetable-based operation in urban transport: run-time optimisation and improvements in the operating process," Transportation Research Part A: Policy and Practice, vol. 45, no. 8, pp. 721-740, 2011.

[36] K. Steiner and S. Irnich, "Schedule-based integrated intercity bus line planning via branch-and-cut," Transportation Science, vol. 52, no. 4, pp. 882-897, 2018.

[37] S. Zhang, A. A. Ceder, and Z. Cao, "Integrated optimization for feeder bus timetabling and procurement scheme with consideration of evironmental impact," Computers and Industrial Engineering, vol. 145, Article ID 106501, 2020.

[38] H. Ma, X. Li, and H. Yu, "Single bus line timetable optimization with big data: a case study in beijing," Information Sciences, vol. 536, pp. 53-66, 2020.

[39] W. Domschke, "Schedule synchronization for public transit networks,” OR Spektrum, vol. 11, no. 1, pp. 17-24, 1989.

[40] A. Ceder, B. Golany, and O. Tal, "Creating bus timetables with maximal synchronization," Transportation Research Part A: Policy and Practice, vol. 35, no. 10, pp. 913-928, 2001.

[41] A. Gong, "A model to create bus timetables to attain maximum synchronization considering waiting times at transfer stops," Master's Thesis, University of South Florida, Tampa, FL, USA, 2004.

[42] Z. Liu, J. Shen, and H. Wang, W. Yang, "Regional bus timetabling model with synchronization," Journal of Transportation Systems Engineering and Information Technology, vol. 7, no. 2, pp. 109-112, 2007.

[43] O. J. Ibarra-Rojas, F. López-Irarragorri, and Y. A. Rios-Solis, "Multiperiod bus timetabling," Transportation Science, vol. 50, no. 3, pp. 805-822, 2016.

[44] O. J. Ibarra-Rojas, R. Giesen, and Y. A. Rios-Solis, "An integrated approach for timetabling and vehicle scheduling problems to analyze the trade-off between level of service and operating costs of transit networks," Transportation Research Part B: Methodological, vol. 70, pp. 35-46, 2014.
[45] O. J. Ibarra-Rojas and Y. A. Rios-Solis, "Synchronization of bus timetabling," Transportation Research Part B: Methodological, vol. 46, no. 5, pp. 599-614, 2012.

[46] R. C. W. Wong, T. W. Y. Yuen, and K. W. Fung, J. M. Y. Leung, "Optimizing timetable synchronization for rail mass transit," Transportation Science, vol. 42, no. 1, pp. 57-69, 2008.

[47] Y. Shafahi and A. Khani, "A practical model for transfer optimization in a transit network: model formulations and solutions," Transportation Research Part A: Policy and Practice, vol. 44, no. 6, pp. 377-389, 2010.

[48] L. Kang, J. Wu, and H. Sun, X. Zhu, B. Wang, "A practical model for last train rescheduling with train delay in urban railway transit networks," Omega, vol. 50, pp. 29-42, 2015.

[49] X. Guo, H. Sun, and J. Wu, J. Jin, J. Zhou, Z. Gao, "Multiperiod-based timetable optimization for metro transit networks," Transportation Research Part B: Methodological, vol. 96, pp. 46-67, 2017.

[50] X. Jin, J. Wu, and J. Zhou, X. Yang, D. Wu, Z. Gao, "First-train timing synchronisation using multi-objective optimisation in urban transit networks," International Journal of Production Research, vol. 57, no. 11, pp. 3522-3537, 2019.

[51] M. Abdolmaleki, N. Masoud, and Y. Yin, "Transit timetable synchronization for transfer time minimization," Transportation Research Part B: Methodological, vol. 131, pp. 143-159, 2020.

[52] Y. Guo, B. Mao, and Y. Bai, T. K. Ho, Z. Li, “Timetable synchronization of last trains for urban rail networks with maximum" accessibility," Transportation Research Part C: Emerging Technologies, vol. 99, pp. 110-129, 2019.

[53] J. P. Fonseca, E. Van Der Hurk, and R. Roberti, A. Larsen, “A matheuristic for transfer synchronization through integrated timetabling and vehicle scheduling," Transportation Research Part B: Methodological, vol. 109, pp. 128-149, 2018.

[54] P. Fouilhoux, O. J. Ibarra-Rojas, and S. Kedad-Sidhoum, Y. A. Rios-Solis, "Valid inequalities for the synchronization bus timetabling problem," European Journal of Operational Research, vol. 251, no. 2, pp. 442-450, 2016.

[55] Y. Shen and J. Xia, "Integrated bus transit scheduling for the Beijing bus group based on a unified mode of operation," International Transactions in Operational Research, vol. 16, no. 2, pp. 227-242, 2009.

[56] M. Hickman, P. Mirchandani, and S. Vob, Computed-aided Systems in Public Transport. Lecture Notes In Economics \& Mathematical Systems, Springer, Berlin, Heidelberg, 2008.

[57] D. PeñaA. Tchernykh et al., "Operating cost and quality of service optimization for multi-vehicle-type timetabling for urban bus systems," Journal of Parallel and Distributed Computing, vol. 133, pp. 272-285, 2019.

[58] L. Wu, H. K. Lo, and F. Xiao, "Mixed bus fleet scheduling under range and refueling constraints," Transportation Research Part C: Emerging Technologies, vol. 104, pp. 443-462, 2019.

[59] L. Bodin and B. Golden, "Classification in vehicle routing and scheduling," Network, vol. 11, no. 1, pp. 97-108, 1981.

[60] L. Bodin, B. Golden, A. Assad et al., "Routing and scheduling of vehicles and crews: the state of the art," Computers and Operations Research, vol. 10, no. 1, pp. 63-212, 1983.

[61] S. Bunte and N. Kliewer, "An overview on vehicle scheduling models,” Public Transport, vol. 1, no. 4, pp. 299-317, 2009.

[62] R. Freling, A. P. M. Wagelmans, and J. M. P. Paixão, "Models and algorithms for single-depot vehicle scheduling," Transportation Science, vol. 35, no. 2, pp. 165-180, 2001.

[63] L. J. J. Van Der Bruggen, J. K. Lenstra, and P. C. Schuur, "Variable-depth search for the single-vehicle pickup and delivery problem with time windows," Transportation Science, vol. 27, no. 3, pp. 298-311, 1993. 
[64] J. Zhang, W. Li, and F. Qiu, "Optimizing single-depot vehicle scheduling problem: fixed-interval model and algorithm," Journal of Intelligent Transportation Systems, vol. 19, no. 3, pp. 215-224, 2015.

[65] O. J. Ibarra-Rojas, F. Delgado, and R. Giesen, J. C. Muñoz, Planning, "operation, and control of bus transport systems: a literature review," Transportation Research Part B: Methodological, vol. 77, pp. 38-75, 2015.

[66] N. Kliewer, T. Mellouli, and L. Suhl, "A time-space network based exact optimization model for multi-depot bus scheduling," European Journal of Operational Research, vol. 175, no. 3, pp. 1616-1627, 2006.

[67] M. Michaelis and A. Schobel, "Integrating line planning, timetabling, and vehicle scheduling: a customer-oriented heuristic," Public Transportation, vol. 1, no. 4, pp. 211-232, 2009.

[68] S. Hassold and A. Ceder, "Public transport vehicle scheduling featuring multiple vehicle types," Transportation Research Part B: Methodological, vol. 67, pp. 129-143, 2014.

[69] V. Schmid and J. F. Ehmke, "Integrated timetabling and vehicle scheduling with balanced departure times," $O R$ Spectrum, vol. 37, no. 4, pp. 903-928, 2015.

[70] J. Li, T. Chen, W. D. Fan et al., "Integrated approach to vehicle scheduling and bus timetabling for an electric bus line," Journal Of Transportation Engineering, Part A: Systems, vol. 146, Article ID 4019073, 2020.

[71] Z. Cao and A. Ceder, "Autonomous shuttle bus service timetabling and vehicle scheduling using skip-stop tactic," Transportation Research Part C: Emerging Technologies, vol. 102, pp. 370-395, 2019.

[72] B. David, M. Kresz, "Multi-depot bus schedule assignment with parking and maintenance constrains for intercity transportation over a planning period," Transportation Letters: The International Journal of Transportation Research, vol. 12, pp. 66-75, 2020.

[73] P. C. Guedes, D. Borenstein, and M. Sâmara Visentini, O. C. B. De Araújo and A. F. Kummer Neto, "Vehicle scheduling problem with loss in bus ridership," Computers \& Operations Research, vol. 111, pp. 230-242, 2019.

[74] R. Freling, D. Huisman, and A. P. M. Wagelmans, "Models and algorithms for integration of vehicle and crew scheduling," Journal of Scheduling, vol. 6, no. 1, pp. 63-85, 2003.

[75] A. Graffi and M. Nonato, "An integrated approach to exurban crew and vehicle scheduling," Lecture Notes in Economic \& Mathematical Systems, Springer, Berlin, Heidelberg, 1999.

[76] R. Borndorfer, A. Lobel, and S. Weider, "A bundle method for integrated multi-depot vehicle and duty scheduling in public transit," Computer-aided Systems in Public Transport.vol. 600, pp. 3-24, 2008.

[77] M. A. Boschetti, A. Mingozzi, and S. Ricciardelli, "An exact algorithm for the simplified multiple depot crew scheduling problem," Annals of Operations Research, vol. 127, no. 1-4, pp. 177-201, 2004.

[78] S. Singh and D. Huisman, "Vehicle and crew scheduling: solving large real-world instances with integrated approach," in Proceedings of the Presented at the 9th International Conference on Computer-Aided Scheduling of Public Transport, San Diego, CA USA, August 2004.

[79] K. Haase, G. Desaulniers, and J. Desrosiers, "Simultaneous vehicle and crew scheduling in urban mass transit systems," Transportation Science, vol. 35, no. 3, pp. 286-303, 2001.

[80] M. Mesquita and A. Paias, "Set partitioning/covering-based approaches for the integrated vehicle and crew scheduling problem," Computers \& Operations Research, vol. 35, no. 5, pp. 1562-1575, 2008.

[81] V. Gintner, I. Steinzen, and L. Suhl, "A time-space network based approach for integrated vehicle and crew scheduling in public transport," in the Proceedings of the EURO World Group on Transportation Joint Conference, Delft, Netherlands, June 2006.

[82] I. Steinzen, V. Gintner, and L. Suhl, N. Kliewer, "A time-space network approach for the integrated vehicle- and crewscheduling problem with multiple depots," Transportation Science, vol. 44, no. 3, pp. 367-382, 2010.

[83] N. Kliewer, B. Amberg, and B. Amberg, "Multiple depot vehicle and crew scheduling with time windows for scheduled trips," Public Transport, vol. 3, no. 3, pp. 213-244, 2012.

[84] H. L. Petersen, A. Larsen, and O. B. G. Madsen, B. Petersen, S. Ropke, "The simultaneous vehicle scheduling and passenger service problem," Transportation Science, vol. 47, no. 4, pp. 603-616, 2013.

[85] C. Gershenson and L. A. Pineda, "Why does public transport not arrive on time? The pervasiveness of equal headway instability," Plos One, vol. 4, no. 10, Article ID e7292, 2009.

[86] S. M. Moosavi, A. Ismail, and C. W. Yuen, "Using simulation model as a tool for analyzing bus service reliability and implementing improvement strategies," PLoS One, vol. 15, no. 5, Article ID e0232799, 2020.

[87] G. Liu and S. C. Wirasinghe, "A simulation model of reliable schedule design for a fixed transit route," Journal of Advanced Transportation, vol. 35, no. 2, pp. 145-174, 2001.

[88] S. C. Wirasinghe and G. Liu, "Optimal schedule design for a transit route with one intermediate time point," Transportation Planning and Technology, vol. 19, no. 2, pp. 121-145, 1995.

[89] Y. Wu, J. Tang, and X. Luo, "Comparative analysis of operation strategies in schedule design for a fixed bus route," International Transactions in Operational Research, vol. 22, no. 3, pp. 545-562, 2015.

[90] Y. Yan, Q. Meng, and S. Wang, X. Guo, "Robust optimization model of schedule design for a fixed bus route," Transportation Research Part C: Emerging Technologies, vol. 25, pp. 113-121, 2012.

[91] N. Van Oort, J. W. Boterman, and R. Van Nes, "The impact of scheduling on service reliability: trip-time determination and holding points in long-headway services," Public Transport, vol. 4, no. 1, pp. 39-56, 2012.

[92] X. Dou, Y. Yan, and X. Guo, "Time control point strategy coupled with transfer coordination in bus schedule design," Journal of Advanced Transportation, vol. 50, no. 7, pp. 1336-1351, 2016.

[93] Y. Wang, Y. Bie, and L. Zhang, "Joint optimization for the locations of time control points and corresponding slack times for a bus route," KSCE Journal of Civil Engineering, vol. 23, no. 1, pp. 411-419, 2019.

[94] B. Varga, T. Kulcsár, and B. Kulcsar, "Optimally combined headway and timetable reliable public transport system," Transportation Research Part C: Emerging Technologies, vol. 92, pp. 1-26, 2018.

[95] Y. Zhu, B. Mao, and Y. Bai, S. Chen, "A bi-level model for single-line rail timetable design with consideration of demand and capacity," Transportation Research Part C: Emerging Technologies, vol. 85, pp. 211-233, 2017.

[96] Y. Zheng, W. Li, and F. Qiu, "A slack arrival strategy to promote flex-route transit services," Transportation Research Part C: Emerging Technologies, vol. 92, pp. 442-455, 2018. 
[97] J. Zhang and X. Du, "A second-order reliability method with first-order efficiency," Journal of Mechanical Design, vol. 132, pp. 973-984, 2010.

[98] Y.-G. Zhao and T. Ono, "A general procedure for first/secondorder reliability method (FORM/SORM)," Structural Safety, vol. 21, no. 2, pp. 95-112, 1999.

[99] C. Cheng, L. Zhang, R. G. Thompson, "Reliability analysis of road networks in disaster waste management," Waste Management, vol. 84, pp. 383-393, 2019. 